\title{
Reviewer acknowledgement Reviews in Chemical Engineering volume 36 (2020)
}

https://doi.org/10.1515/revce-2020-0099

The editors and the publisher wish to thank the following colleagues for their kind assistance in acting as referees for the journal this year: ${ }^{1}$

Abbas, Syed Zaheer

Agarwal, V. K.

Agrawal, Dinesh

Alekseenko, Sergey V.

Al-Othman, Zeid Abdullah

Alsheri, Sultan

Ansari, Amir

Ardekani, Mehdi Niazi

Babamohammadi, Shervan

Baldea, Michael

Bao, Shenxu

Barnea, Dvora

Basavaraj, Madivala G.

Bassin, João Paulo

Baudu, Michel

Bechelany, Mikhael

Benamor, Abdelbaki

Bengoa, Fernando

Berti, Federico

Bhattacharya, Proma

Blais, Bruno

Bollinger, Jean-Claude

Bonn, Daniel

Boutilier, Michael

Bradford, Michael

Brus, Grzegorz

Buder, Irmgard

Burghelea, Teo

Calinescu, Ioan

Carreon, Moises A.

Carrillo Pedroz, Raul

Chen, Lin

Chen, Xi

Chew, Jia Wei

Chhabra, Rajendra P.

Chibane, Lemnouer
Chung, Neal Tai-Shung

Clegg, John R.

Costa, Pedro

Coville, Neil

Criscuoli, Alessandra

Dash, Sukanta Kumar

De Wilde, Juray

Dejam, Morteza

Deng, Wen-yi

Dharmalingam, S.

Dimakopoulos, Yannis

Divoux, Thibaut

Dixon, David

Duan, Lunbo

Dzhardimalieva, Gulzhian I.

Edgar, Thomas F.

Etchells, Arthur

Evans, Drew

Everson, Raymond C.

Fan, Mizi

Fanaei, Mohammad A.

Feldman, Yuri

Foerst, Petra

Gallucci, Fausto

Garg, Sahil

Giagnorio, Mattia

Goikhman, Roman

Gómez-Pastora, Jenifer

Gorte, Ray

Gotor Fernández, Vicente

Grützner, Thomas

Habashi, Fathi

Han, You
Hassan, P. A.

Hatton, Alan

He, Hongping

He, Yucai

Hristov, Jordan

Huang, Caoxing

Huang, K.

Hüner, Ümit

Izak, Pavel

Jafariesfad, Narjes

Jiang, Chang Zhong

Jiang, Fujie

Jin, Ren-Cun J.

Kaewpirom, Supranee

Kaippamangalath, Nimisha

Kalal, Sid

Khinast, Johannes

Kim, Seung Wook

Klinzing, George

Kopecký, Dušan

Koponen, Antti I.

Kord, Shahin

Korin, Eli

Kost, Joseph

Kumar, Vineet

Landau, Miron

Lasala, Silvia

Leal, L. Gary

Lee, Jang Yong

Legrand, Jack

Li, Huazheng

Li, Hui-quan

Li, Jinghai

Li, Jin-lin

1 The list covers the time span October 9, 2019 to November 3, 2020. 
Li, Yingcheng

Liné, Alain

Liu, Chengchao

Liu, Jian

Liu, Mingyan

Liu, Xuguang

Liu, Y. L.

Liu, Zhong-Wen

Lonyi, Ferenc

Lopes, Andre Miguel

Ma, Hongwen

Ma, Wenping

Maleki, Ali

Mamane, Hadas

Marin, Luminita

Marti, Jordi

Martinez-Huitle, Carlos

Martirosyan, Karen

Masunov, Artem

Merino-Garcia, Ivan

Milestone, Neil B.

Mofarahi, Masoud

Moghanloo, Rouzbeh G.

Mokhtari, Mehdi

Moulik, Siddhartha

Munshi, Basudeb

Naderi, Hojat

Narsimhan, Ganesan

Nasir, Rizwan

Nasrifar, Khashayar

Nguyen, Van Khanh

Nigar, Hakan

Odey, Emmanuel

Ortiz, Inmaculada

Osgouei, Reza

Pang, Xu-Ming

Papadopoulos, Athanasios I.

Passalacqua, Enza

Pech-Canul, Martin I.

Pellegrini, Laura

Polishuk, Ilya
Qin, Chao

Qin, Guotong

Ramírez-Muñoz, Jorge

Raquez, Jean-Marie

Riahi, Siavash

Ribeiro, Ana P. C.

Rojas, Sergio

Roose-Amsaleg, Celine

Rubio, Ramón G.

Rytter, Erling

Samanta, Amar Nath

Schlosser, Štefan

Schroeder, Avi

Sebastian, Victor

Sharif, Alireza

Shaulsky, Evyatar

Shen, Shufeng

Shen, Y. S.

Shtern, Vladimir N.

Shui, Guan

Singh, Ram Lakhan

Song, Zhanlong

Soum-Glaude, Audrey

Stefanidis, Georgios D.

$\mathrm{Su}$, Jincai

$\mathrm{Su}$, Jun-Feng

Sun, Jie

Suzuki, Kosuke

Taghikhani, Vahid

Tan, Xiaoyao

Taylor, Ross

Tian, Zhou

Toth, Andras Jozsef

Trabold, Thomas A.

Tsakoumis, Nikolaos

Ullmann, Amos

Vaimakis, Tiverios

Virolainen, Sami

Wang, Baodong

Wang, Chengbing
Wang, Cong

Wang, Li

Wang, Moran

Wang, Shao-Wei

Wang, Shixing

Wang, $Z$.

Ward, Jeffrey

Wei, Qufu

Winter, Gerhard

$\mathrm{Wu}$, Zhansheng

Wyszynski, Miroslaw

$\mathrm{Xu}$, Chengyuan

Yang, Jingshuai

Yang, Yuechao

Ye, Guang-Hua

Zagoruiko, Andrey N.

Zamani, Yahya

Zaragoza, Guillermo

Zeini, Abbas

Zeng, Gaofeng

Zhang, Huan

Zhang, Jin

Zhang, Tao

Zhang, Wengang

Zhang, Xiao

Zhang, Xunli

Zhang, Yanfeng

Zhang, Ying

Zhao, Donglin

Zhao, Lingling

Zhao, Tian-Sheng

Zhao, Xin

Zheng, Junsheng

Zhong, Liangshu

Zhong, Wenqi

Zhou, Jia-Wei

Zilberman, Meital

Zivkovic, Vladimir

Zucker, Ines

Zuo, Shufeng 